\title{
A Design review of Smart Stick for the Blind Equipped with Obstacle Detection and Identification using Artificial Intelligence
}

\author{
Balu N. Ilag \\ $\mathrm{Al}$ and UC \& Collaboration Expert \\ Silicon Valley, CA
}

\author{
Yogesh Athave \\ Sr. Software Engineer \\ Silicon Valley, CA
}

\begin{abstract}
Visually impaired individuals find it more challenging to move about independently because of their compromised vision. Moreover, a blind person's capacity to navigate in a given setting, along with their ability to organize their daily activities are vital to their health and wellbeing. Organizing any commonplace activity can be especially difficult for a blind man/woman if he/she has not learned to distinguish between different items like drug containers and packaged goods, just by feeling with the hands. The more saddening fact is that there are tens of millions of visually impaired persons worldwide who have to go through such experience and are dependent on others for their wellbeing and happiness. The encouraging news, however, is that the rapid advancement in technology has seen the innovation of better systems for assisting the disabled, including the blind, such as the AI glasses, which can provide intelligent navigation capabilities to the blind. This paper reviews the design of a smart cane, i.e., A smart stick for the blind, equipped with obstacle recognition using AI Technologies adds more virtual visibility in their journey. It shows that such a stick can be a significant boon to the blind.
\end{abstract}

\section{General Terms}

Your general terms must be any term which can be used for general classification of the submitted material such as Pattern Recognition, Security, Algorithms et. al.

\section{Keywords}

Unified Communication System (UCS), UC application.

\section{INTRODUCTION}

Vision, to begin with, is the most crucial part of human psychology, considering that $83 \%$ of the information that a person obtains from the environment is through the sight (Elmannai \& Elleithy 565). However, there are many people with serious visual impairments that prevent them from traveling individually. According to a 2018 report by the World Health Organization (WHO), there are roughly 1.3 billion people in the world who are visually impaired, 39 million of whom are blind, and 246 million have low vision. Accordingly, these individuals must use a range of techniques and tools to aid them in movement. Some of the oldest aids for the visually impaired have been the walking cane, also known as the white stick, and the guide dog. Though useful, these aids have significant setbacks, as will be discussed later in this paper (Shoval, Ulrich, \& Borenstein 9). The rapid advancement of modern technology has, however, seen the advent of better systems such as the assisted vision smart glasses and the smart cane that can provide intelligent navigation capabilities to the blind. This paper reviews the design of a smart cane, i.e., an electronic supplementary eye stick, equipped with image recognition technologies that capture front, side, and backend images and use AI and machine learning to process them.

The smart cane is an alternative to the common/traditional walking stick, which is a purely mechanical device used to detect ground obstacles, including holes, steps, uneven surfaces, and other things that may pose a danger. The traditional white cane is inexpensive and very lightweight and small, which makes it foldable able to fit in a pocket. Though useful, the traditional white cane has some critical setbacks, including the fact it takes hard training for one to be able to use them effectively. This is a significant "hidden" cost. Further, it only conveys a limited amount of information and allows a limited range of motion because the user can only scan the small area ahead of him/her and objects can only be detected through contact. This can be inconvenient to a traveler and those around him/her, for instance, if one is traveling in a crowded street (Shoval et al. 10). In the case of guide dogs, albeit these can be capable guides for the blind, they need extensive training. Moreover, a fully trained guide dog can cost anywhere from $\$ 12,000$ to $\$ 20,000$, which is quite high, considering that their useful life is only about five years. Shoval et al. further explain that many visually impaired persons are elderly and find it very difficult to provide appropriate nurturance to another living being. Thus, only about $1 \%$ of visually impaired persons in Australia have guide dogs (10).

The advent of ultrasonic and radar technologies, however, have seen the introduction of electronic travel aids (ETAs) for the blind over the past 40 years. In regard to working principles, most ETAs function much the same way as a radar system, i.e., an ultrasonic "beam" or a laser is emitted in a given direction in space, and the beam is reflected from the objects it meets along the way. A matching sensor receives the reflected beam, determines the distance to the object conveys this information to a user via tactile or audible signals. Most ETAs can identify objects that are up to 16 feet $(5 \mathrm{~m})$ away from the user albeit they need continuous scanning of the environment in the desired direction (Shoval et al. 8). The smart cane is a boon for the blind, in that it combines the power of a microcontroller, i.e., a small lowcost single-chip computer that can perform automatic calculations at high speeds and with remarkable accuracy, with an ultrasonic wave receiver and transmitter to measure distances from objects and allow the blind to navigate places with much ease (Shoval et al. 8).

\section{A REVIEW OF RELATED WORKS}

So far researchers have done significant work for the blind and visually impaired by developing many different kinds of assistive technologies to aid them in navigation. Some of the different designs of the smart cane are discussed below along with their modes of operation. 


\subsection{Koley and Mishra's "voice-operated outdoor navigation system for the visually impaired."}

This version of the smart cane uses a sick fitted with ultrasonic sensors, an audio output system, and a GPS module. The GPS module has a memory chip used to save different locations. The user can set the location via voice, and the GPS will guide him/her to that location. This system also gives the speed of motion, along with the distance left to reach the destination. The ultrasonic sensors will trigger the cautioning voice whenever they detect an obstacle along the path. Somnath and Ravi's smart cane is technically a low-cost advanced navigation tool for the blind. Its voice guide has remarkable accuracy. The device uses the AR processor, which has a substantial memory space that allows for highspeed operation. The one drawback for the cane, however, is that it cannot be used indoors since the GPS signal will be lost. Moreover, the precision of the GPS signal requires improvement as it is only controllable within a 16 feet radius. Also, users have to undergo thorough training before they can use the system effectively (Koley and Mishra 153).

\subsection{GPS Artificial Vision Assistance}

The GPS artificial vision assistance was developed by Shruti Kumbhare and A. Sakhore. The device features object detections, real-time assistance via GPS, artificial vision, and a voice circuit. A camera is fitted on the blind user's head, which will employ algorithms to identify obstacles in front of him/her. Ultrasonic sensors also assist with object detection. The GPS guides the user to his/her destination. One strength of this system is that precision of the artificial unit affords the user with high output accuracy. The gadget's drawback, however, is that its design is highly complex, which makes it difficult to operate (Kumbhare \& Sakhore).

\subsection{Sakhardande, Pattanayak, and Bhowmick's smart cane}

Sakhardande et al. designed a smart cane that uses normal ultrasonic sensors and the ATMEL microcontroller. The cane's power is supplied by two rechargeable batteries and recharging can be done through an AC adopter or a USB cable. When the system detects an obstacle/object, the controller triggers a buzzer and vibrations to alert the user. Sakhardande et al.'s smart cane has a range of three meters. One strength of this system is that it is quite simple to use. Further, the rechargeable batteries eliminate the need for regular replacements. The system is also easily packable in a small package. The downside, however, is that this system is unidirectional and is low in accuracy (1262).

\subsection{The C5 laser cane}

The C5 Laser Cane was developed by Malvern Benjamin, Jr., Nazir A. Ali, and Adamo F. Schepis. It is based on optical triangulation with three photodiodes as receivers and three transmitters. A laser beam is aimed ahead, and above and after colliding with an obstacle, it is reflected back and acquired by three photodiodes which function as receivers. The UPchannel pinpoints/detects objects at head-height; whereas the FORWARD channel identifies obstacles from the cane's tip forward in the range of 5 to 11 feet (i.e., 1.5-3.5 m). The DOWN channel, on the other hand, detects drop-offs to the user's front. A high pitch beep via the microphone indicates object detection. The drawback of this gadget, however, is that it offers the user, not technical assistance in regard to the direction to take after the object is detected (Benjamin et al. $54 \mathrm{NOV})$.

\subsection{The Intelligent Guide Stick}

This device was developed by Sung Kang, Young Kim, and Hyuk Moon in 2001. It comprises an ultrasound displacement sensor, a microcontroller, and two DC motors, with a total weight of 4kgs. The guide stick's height and width are 85 and $24 \mathrm{~cm}$ respectively. The system's AI can detect the exact position of an object and relate that information to the user. Detection entails a "map-matching technique" that involves the ultrasonic sensors, the DC motors, and the controller that connects to an encoder. When the rotators turn 18 degrees, the IR sensors that are attached to both wheels will convey the signal to the CPU to give a location update. This way the intelligent guide stick is incredibly accurate in detection and can provide continuous updates about obstacle position. The downside of this gadget, however, is that it is highly complex and costly. Moreover, the $4 \mathrm{~kg}$ weight makes it quite heavy as compared to the other systems (Kang et al. 3208).

\subsection{The Smart Stick}

The smart stick was designed by Jacio Jose, Miguel Farrajota, Jose Rodrigues, and Hans du Baf in 2011. It is a cheap, smallsized, wearable navigation aid. The stick uses GPS to guide the user to a destination, along with local navigation to negotiate paths, corridors, and sidewalks and enables the user to avoid still, as well as mobile obstacles. The device also features a stereo camera that is worn at chest height, along with a portable computer in a shoulder strapped pocket and a small speaker or an earphone. The whole system when in use is inconspicuous, which reduces disruptions while working (Jose et al. 2).

\subsection{The Guide-Cane}

The guide-cane operates much like the white cane, but the difference is that it rolls on wheels that hold the guide-cane's weight during normal operation. It consists of a servomotor, a built-in computer, ten ultrasonic sensors, and a min joystick for control. The servomotor can steer the wheel right and left relative to the cane. When an object is detected, the wheel shifts to the opposite side alerting the user to do likewise. This way the guide-cane allows faster navigation. Both wheels are fitted with encoders to determine their relative motion. The computer chooses a heading and turns the wheel accordingly based on the sensor data from its sonars and user input (Borenstein \& Ulrich 10).

Other existing devices for the blind include:

\subsection{The assisted vision smart glasses}

The assisted vision smart glasses were designed to aid individuals with very low vision to navigate unfamiliar settings, recognize obstacles, and achieve a higher degree of independence. The glasses are based on the fact that most blind individuals have at least some dim vision. The assisted vision smart glasses are intended to capitalize on this sight. The glasses are made of OLED displays, with a gyroscope, a GPS module, an earpiece, a compass, and two small cameras. Incoming data is processed, and then used in a myriad of ways, for instance, brilliance can be used to show depth. Since most visually impaired persons can distinguish brilliance from dullness, the glasses can brighten anything close to the wearer so that they can discern obstacles and persons. The GPS module can provide directions, and the gyroscope assists the glasses in calculating perspective changes as the wearer moves. The camera can also work with the computing module to help read markers along the way (cit). 


\subsection{The AI glasses}

AI glasses integrate a host of features, including artificial intelligence, ultrasound techniques, and computational geometry to create an essential aid for visually impaired persons. By linking glasses with GPS technology of a tablet, along with stereo sound sensors, the prototype can issue spoken commands, recognize currency denominations, read signs, etc. The estimated cost of AI glasses is between $\$ 1000$ and $\$ 1500$ (cit)

\subsection{The ARIANNA app}

The Arianna app is the pAth Recognition for Indoor Assisted NavigatioN with Augmented Perception, which allows blind persons to navigate indoor environments with the help of a smartphone. Colored tape is stuck on the group to mark out particular routes. A user then points his/her smartphone to the ground. If he/she finds the line, the phone will vibrate. So, there are no audio interferences, and users can use the device with no headphones (cit).

Object/obstacle detection and recognition is a critical component for understanding a scene. Elmannai and Elleithy elaborate that the human visual system is fast, robust, selective, and powerful. High selectivity allows us to tell differences between identical objects, like the faces of identical twins. Robustness allows us to categorize objects of the same class despite notwithstanding significance variances in aspects such as color, occlusion, illumination, etc. In fact, studies have found that the human system can discriminate among thousands of different object groups. What is more is that our object recognition processes is very fast, taking as little as 100 to $200 \mathrm{~ms}$. (565). Shoval et al., however, point out that it is very hard to develop selective and robust computer visual algorithms for the recognition of objects that are identical or those with large variations. For instance, the stateof-the-art detection techniques necessitate many training examples, even thousands, and very long periods to learn the visual models of one object class (11).

\section{SMART STICK FOR THE BLIND EQUIPPED WITH OBSTACLE DETECTION AND IDENTIFICATION}

The smart cane for this project is an electronic supplementary eye stick, equipped with image capturing and recognition using Artificial Intelligence technologies. In this regard, it will have the following components:

\subsection{A Raspberry PI}

The Raspberry PI 3 is a microcontroller board n PI series. It can be considered as a single board computer that works on LINUX operating system. According to a 2018 article, the board not only has tons of features it also has terrific processing speed making it suitable for advanced applications. here wireless connectivity is needed. Raspberry PI 3 has wireless LAN and Bluetooth facility by which you can setup WIFI HOTSPOT for internet connectivity. Raspberry PI 3 had dedicated port for connecting touch LCD display which is a feature that completely omits the need of monitor. Raspberry PI 3 also has dedicated camera port so one can connect camera without any hassle to the PI board. Take the 16GB micro SD card and dedicate it specifically for PI OS. Board has a reset button, and a power jack. With an operating voltage of $5 \mathrm{~V}$, the tool is highly user-friendly. It can be connected to a computer directly through a USB cable or powered up with a battery, or an AC-DC adapter. The Raspberry PI 3 will also contain the Smart Cane app with the AI-based algorithm output tags for image tagging based on context, content, and the actions identified in the image. Image tagging with the Smart Cane app will involve sending an authorized web request to the subscription endpoint and observing the information returned in the standard JavaScript Object Notation (JSON) format.

\subsection{Ultrasonic sensors}

The smart stick will have four HC-SRC04 ultrasonic sensors, one of which will detect obstacles in front of the person, and three of which will be used to detect obstacles on the stick's sides. An ultrasonic sensor is a sensor that detects objects using sound waves. As pointed out earlier, it works the same way as sonar or radar works. High-frequency sound waves are generated and received back, and sensors determine the distance from the time it takes to send and receive the echo signals. An HC-SRC04 ultrasonic sensor contains four pins ground, Vcc, trigger, and echo. It has a range of 2 to $400 \mathrm{~cm}$. Usually, it has two openings; one for the transmitter and the other for the receiver. (Vanitha, Rajiv, Elangovan, \& Kumar 3).

\subsection{Raspberry Pi Camera Module}

The Raspberry Pi Camera Module will be used, Camera has 8 Megapixels with 1080p30, 720p60 video mode and Camera had $3280 \times 2464$ pixels resolution. Their purpose will be to capture images which will be used to identify objects. One camera will be placed at the front end of the stick to detect and capture the object, which will be transferred to the microcontroller via Bluetooth or wireless network. The microcontroller will send an authorized web request to a subscription endpoint for identification. Object identification through the images will be done via image tagging at the application end.

\subsection{A voice playback module}

This module will inform the user of the identified obstacle through a microphone/a pair of headphones. The WTV-SR IC recognition module will be used for this project. It can record and fix voice playback. It offers a variety of control modes and is tremendously advantageous in terms of cost and efficiency (Vanitha et al. 3).

\subsection{The GPS/GSM module}

The GPS module has a memory chip that will be used to save different locations. The user can set the location via voice, and the GPS will guide him/her to that location. The GSM modem will be used to send a message to all the saved numbers in the system in case the user issues an emergency alert call. 


\section{SYSTEM DESIGN}

Figure shows design diagram and high-level flow.

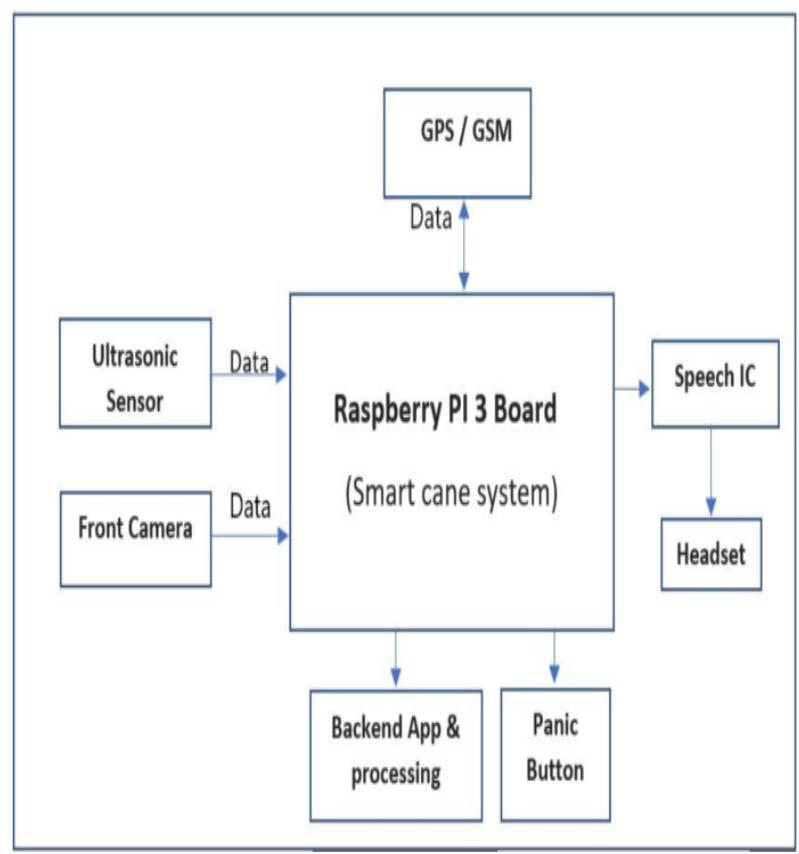

Fig 1: Block Diagram

Here are the steps involved in image detection and identification. The Raspberry PI board is the main part of the system. It controls the other system components. When one of the ultrasonic sensors picks any object within a 180-degree path, it will send the information to the microcontroller, including the bearing and distance from the blind user. The microcontroller, in turn, will issue a command through to the appropriate camera to take a photograph of the object. The digital image will be sent back to the controller, where the Smart Cane app which will send an authorized web request to a subscription endpoint for identification. Object identification through the images will be done via image tagging at the endpoint based on context, content, and the actions identified in the image. The information about the object in the image will be returned in the standard JavaScript Object Notation (JSON) format and be conveyed to the user via a microphone/a pair of headphones. This model is based on Sathya et al.'s (4531) smart walking stick model.

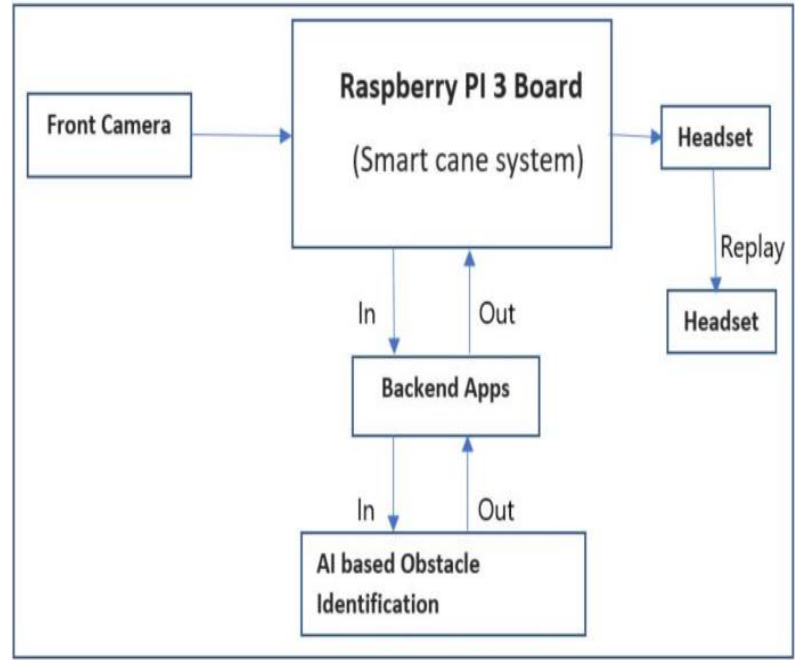

Fig. 2. Obstacle transfer process
The Operation of the AI System:

\subsection{Obstacle Tagging and Recognition}

The smart cane system uses specific features/applications that help in analyzing object details to provide a more accurate identification. For image tagging and recognition, the cane's AI system uses the Describe Image Task/method that returns both a description and a set of tags for every image that is analyzed. The Describe Image method is ideal for situations where image identification requires a set of tags and their description. It works much the same way as the Analyze Image Task/Method, with the only difference being that the endpoint task is "/describe" as rather than "/analyze" (Pissaloux and Velazquez 496).

\subsection{Image Color Identification}

The smart stick also identifies the colors in an image using AI algorithm. This task entails the analysis of three areas/contexts, i.e., the foreground, the background, and the entire image (i.e., dominant foreground and background colors). The system usually analyzes the most "eye-popping" colors to allow the blind individual get a better conception of the object. Moreover, the system uses a Color feature that returns a simple true/false value to indicate to the user if the image is in black and white or not (Rutkowski et al. 648).

\subsection{Image identification via image matching}

The Image Identification feature in the cane's AI system uses/instructs the Analyze Image Method to detect human faces, buildings, type of roads, traffic signals, vehicle coordinates, etc., which correspond to geometric rectangular coordinates for the same, i.e., faces, buildings, roads, traffic signals, vehicle, etc. For an image with a car, for instance, a rectangle is drawn over the car, to identify it (Rutkowski et al. 648). In essence, the blending of all these aspects, i.e., image tagging, description, color identification, and identification via matching will significantly aid in performing detailed image identification and description to aid the blind user in navigation.

Moreover, the GPS and GSM modems will be used to track the user. When the GSM modem gets a message, it will send it to the microcontroller to find the stick's location from the GPS modem and send it back to the GSM modem to respond to the sender. In the event of an emergency, the stick's user will press the emergency button, and a signal will be sent from the GPS modem to the microcontroller and from there to the GSM modem, which will disseminate a message to every saved number in the system. This monitoring system is based on Kumbhare and Sakhore's "smart stick for blind obstacle detection, artificial vision and real time assistance via GPS." 
Table 1: System deficiencies and Remediation

\begin{tabular}{|c|c|}
\hline System Deficiency & Remediation \\
\hline $\begin{array}{c}\text { One drawback for this system } \\
\text { is that it is highly complex } \\
\text { and requires a considerable } \\
\text { amount of practice to be fit to } \\
\text { use it efficiently (Sathya et al. } \\
\text { 4535). }\end{array}$ & $\begin{array}{c}\text { The solution to this problem is } \\
\text { to offer regular training } \\
\text { sessions on its use to the blind } \\
\text { persons (Sathya et al. 4535). }\end{array}$ \\
\hline $\begin{array}{c}\text { Secondly, the numerous } \\
\text { information processing } \\
\text { procedures can significantly } \\
\text { reduce one's speed as he/she } \\
\text { waits for directions (Sathya et } \\
\text { al. 4535). }\end{array}$ & $\begin{array}{c}\text { The solution is to incorporate } \\
\text { the latest information } \\
\text { processing systems which are } \\
\text { a lot faster and will } \\
\text { significantly reduce } \\
\text { processing time (Sathya et al. } \\
\text { 4535). }\end{array}$ \\
$\begin{array}{c}\text { Thirdly, is that this system } \\
\text { may not be accessible for use } \\
\text { in underdeveloped nations due } \\
\text { to reasons such as cost among } \\
\text { other things (Nada, Mashelly, } \\
\text { Fakhr, \& Seddik 7). }\end{array}$ & $\begin{array}{c}\text { A solution to this problem is } \\
\text { subsidizing the production of } \\
\text { gadgets that are intended for } \\
\text { export to developing nations. } \\
\text { Also, funds can also be raised } \\
\text { through charity organizations } \\
\text { for the same purpose (Nada et }\end{array}$ \\
al. 7).
\end{tabular}

\subsection{Experiment and result}

Obstacle images got captured by front motion camera and captured Information sent to backend application and then backend application talks to the Artificial intelligent based system to categorize this information and generate most accurate description, as form of audio file resend raspberry pia and replay obstacle description to visually impaired person.

\section{Analysis:}

1. Time takes to walk one block is 5 mins $(1$ block $=$ 660 feet).

2. Number of Images captured in 5 mins are 20 images.

3. Number of images captured in every minute are 4 images.

4. Per image capture to processing take 1 second means four images capture to processing takes 4 seconds time.

5. Image processing time (each image $)=20$ images / 20 seconds

Following table precisely show obstacle capturing and identification:
Obstacle log Table: 1.1

\begin{tabular}{|c|c|c|}
\hline $\begin{array}{c}\text { Walking Distance } \\
\text { [1 Block] }\end{array}$ & $\begin{array}{c}\text { Obstacle } \\
\text { Category }\end{array}$ & Duration to process \\
\hline 660 feet & 5 cars & 5 seconds \\
\hline & 1 motor cycle & 1 seconds \\
\hline & 6 houses & 6 seconds \\
\hline & 2 women & 2 seconds \\
\hline & 3 men & 3 seconds \\
\hline & $\begin{array}{c}1 \text { traffic } \\
\text { signal }\end{array}$ & 1 seconds \\
\hline 660 feet distance & 20 obstacles & 20 seconds \\
\hline
\end{tabular}

\section{CONCLUSION}

In conclusion, the advent of ultrasonic and radar technologies have brought about significant developments in the world, and most. Researchers have done commendable work in developing many different kinds of assistive technologies to assist the disabled, and more so the blind and visually impaired to become more independent. The smart cane assists the blind to perform navigation and do their work easily by and comfortably. The ordinary blind stick cannot detect obstacles that are out of range of contact, which makes it highly inefficient. Moreover, the user cannot tell the kind of object in front of him nor his distance from it. As for the smart walking stick, objects are not only detected by ultrasound sensors but are also identified through a camera system, and the user is notified accordingly. In this sense, the user will know what is before him/her. Thus, the smart cane is a boon for the blind. It is a masterpiece of technology that has revolutionized the care for the blind. Its relatively low cost implies that soon, many even in developing nations will also be able to access them. Hopefully, even better technologies will be developed to alleviate the hardships and pains that some may be forced to endure in life to a greater extent.

\section{REFERENCES}

[1] Benjamin, J. Malvern, Nazir A. Ali, and Adamo F. Schepis. "A Laser Cane for the Blind." Proceedings of the San Diego Biomedical Symposium, vol. 12, 2011, pp. 53-57.

[2] Borenstein, Johann \& Iwan Ulrich. "The GuideCane-A Computerized Travel Aid for the Active Guidance of Blind Pedestrians." Proceedings of the IEEE International Conference on Robotics \& Automation, Albuquerque, NM, 21-27 April 1997, pp. 1283-1288.

[3] Dhanuja, R., F. Farhana, \& G. Savitha. "Smart Blind Stick Using Arduino." International Research Journal of Engineering and Technology, vol. 5, no. 3, 2008, pp. 2553-2555.

[4] Elmannai, Wafa \& Khaled Elleithy. "Sensor-Based Assistive Devices for Visually-Impaired People: Current Status, Challenges, and Future Directions." Sensors (Basel), vol. 17, no. 3, 2017, pp. 565.

[5] Jose, Jacio, Miguel Farrajota, Joae Rodrigues, and Hans du Baf. "The Smart Vision Local Navigation Aid for the 
Blind and Visually Impaired Persons." International Journal if Digital Content Technology, vol. 5, 2011, pp. $1-7$.

[6] Kang, S. Jae, Young H. Kim, \& In H. Moon "Development of an Intelligent Guide Stick for the Blind." Proceedings of the 2001 IEEE International Conference on Robotics \& Automation, Seoul, Korea, 21-26 May 2001.

[7] Koley, Somnath \& Ravi Mishra. "Voice Operated Outdoor Navigation System for Visually Impaired Persons." International Journal of Engineering Trends and Technology, vol. 3, no. 2, 2012, pp. 153-157.

[8] Kumbhare, Shruti \& A. Sakhore. "A Smart Stick for Blind Obstacle Detection, Artificial Vision and Real Time Assistance via GPS." International Journal of Computers, vol. 7, 2011.

[9] Nada, Ayat, Samia Mashelly, Mahmoud A. Fakhr, and Ahmed F. Seddik. "Effective Fast Response Smart Stick for Blind People." Second International Conference on Advances in Bio-Informatics and Environmental Engineering - ICABEE, At Italy, 2015.

[10] Pissaloux, Edwige and Ramiro Velazquez (Eds.). Mobility of Visually Impaired People: Fundamentals and ICT Assistive Technologies. New York, NY: Springer, 2018.

[11] Rutkowski, Leszek, Marcin Korytkowski, Rafal Scherer, Ryszard Tadeusiewicz, Lotfi A. Zadeh, and Jacek M. Zurada. "Artificial Intelligence and Soft Computing." 11th International Conference, ICAISC 2012, 2012 Zakopane, Poland, April 29-May 3, 2012, Proceedings, Part 1.

[12] Sakhardande, Jayant, Pratik Pattanayak, Mita Bhowmick. "Smart Cane Assisted Mobility for the Visually Impaired." International Journal of Electrical and Computer Engineering, vol. 6, no. 10, 2012, pp. 9-20.

[13] Sathya, D. S. Nithyaroopa, P. Betty, G. Santhoshni, S. Sabharinath, \& M. J. Ahanaa. "Smart Walking Stick for Blind Person." International Journal of Pure and Applied Mathematics, vol. 118, no. 20, 2018, pp. 4531-4536.

[14] Shoval, Shraga, Iwan Ulrich, \& Johann Borenstein. "NavBelt and Guide-Cane: Robotics-Based ObstacleAvoidance Systems for the Blind and Visually Impaired." Special Issue on Robotics in Bio-Engineering, vol. 10, no. 1, 2003, pp. 9-20.

[15] Vanitha, M., A. Rajiv, K. Elangovan, \& S. Vinoth Kumar. "A Smart Walking Stick for Visually Impaired." International Journal of Pure and Applied Mathematics, vol. 119 , no. 16,2018 , pp. 3485-3489.

[16] World Health Organization (WHO). "Blindness and Vision Impairment." WHO, 11 October 2018, https://www.who.int/news-room/factsheets/detail/blindness-and-visual-impairment

[17] Raspberry $\mathrm{Pi}$ information, 26 April 2018, https://components101.com/microcontrollers/raspberrypi-3-pinout-features-datasheet.

[18] https://en.m.wikipedia.org/wiki/City_block, Wikipedia 